\title{
Erratum to: NFAT5-mediated CACNA1C expression is critical for cardiac electrophysiological development and maturation
}

\author{
Wei Li ${ }^{1}$ • Nai-Zhong Zheng ${ }^{2}$ • Qi Yuan ${ }^{3} \cdot \mathrm{Ke} \mathrm{Xu}^{1}$ • Fan Yang ${ }^{1} \cdot \mathrm{Lei} \mathrm{Gu}^{3}$ • \\ Gu-Yan Zheng ${ }^{1}$ - Guo-Jie Luo ${ }^{4}$. Chun Fan ${ }^{5}$ - Guang-Ju Ji ${ }^{3} \cdot$ Bo Zhang ${ }^{2}$. \\ Huiqing Cao ${ }^{1} \cdot$ Xiao-Li Tian ${ }^{1}$
}

Published online: 12 August 2016

(C) Springer-Verlag Berlin Heidelberg 2016

\section{Erratum to: J MolMed}

DOI 10.1007/s00109-016-1444-x

The original version of this article unfortunately contains a layout mistake; the axis labels in Figure 4 are not properly placed.

The correct figure 4 is shown below:

The online version of the original article can be found at http:// doi:10.1007/s00109-016-1444-x.

Huiqing Cao

caohuiqing@pku.edu.cn

$\triangle$ Xiao-Li Tian

tianxiaoli@pku.edu.cn

1 Department of Human Population Genetics, Institute of Molecular Medicine, Peking University, 5 Yiheyuan Road, Beijing 100871, China

2 Key Laboratory of Cell Proliferation and Differentiation of the Ministry of Education, College of Life Sciences, Peking University, 5 Yiheyuan Road, Beijing 100871, China

3 National Laboratory of Biomacromolecules, Institute of Biophysics, Chinese Academy of Sciences, 15 Datun Road, Chaoyang District, Beijing 100101, China

4 School of Electronics Engineering and Computer Science, Peking University, 5 Yiheyuan Road, Beijing 100871, China

5 Department of Biomedical Engineering, Lerner Research Institute, The Cleveland Clinic, 9500 Euclid Avenue, Cleveland, OH 44195, USA 
A
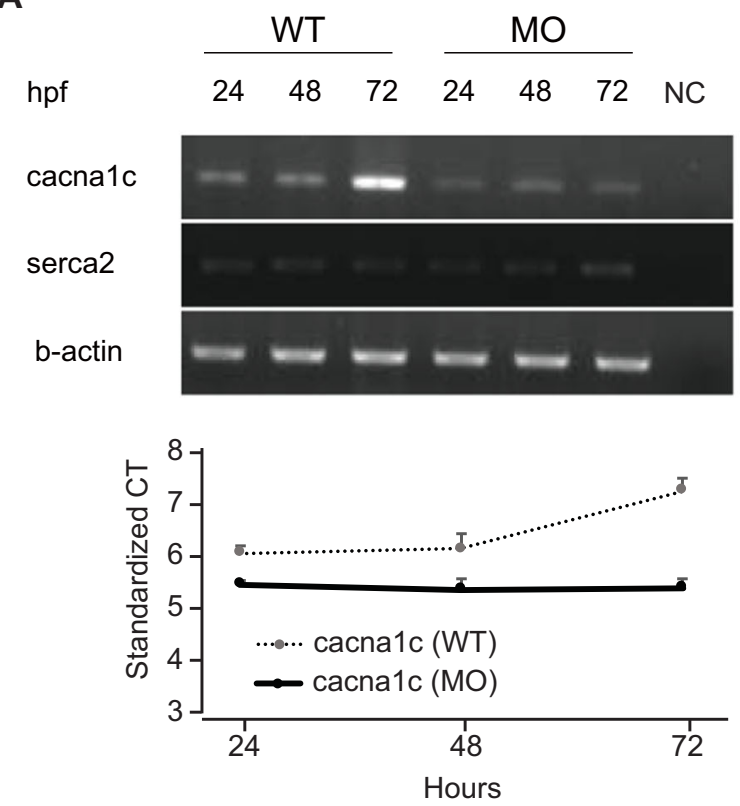

C WT

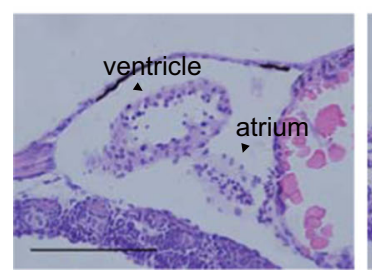

cacna1c-injected

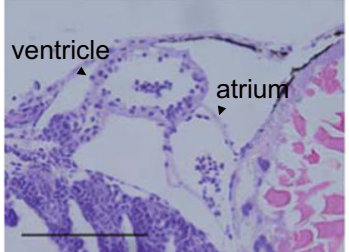

B WT $72 \mathrm{~h}$

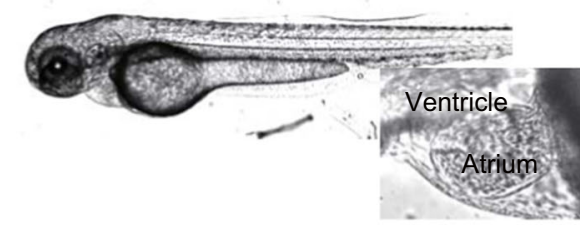

MO $72 \mathrm{~h}$

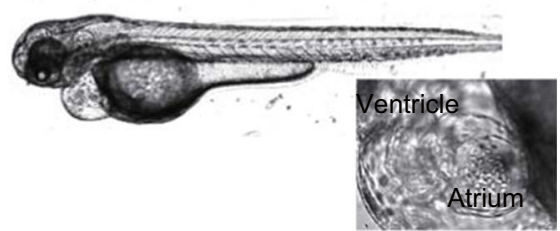

D

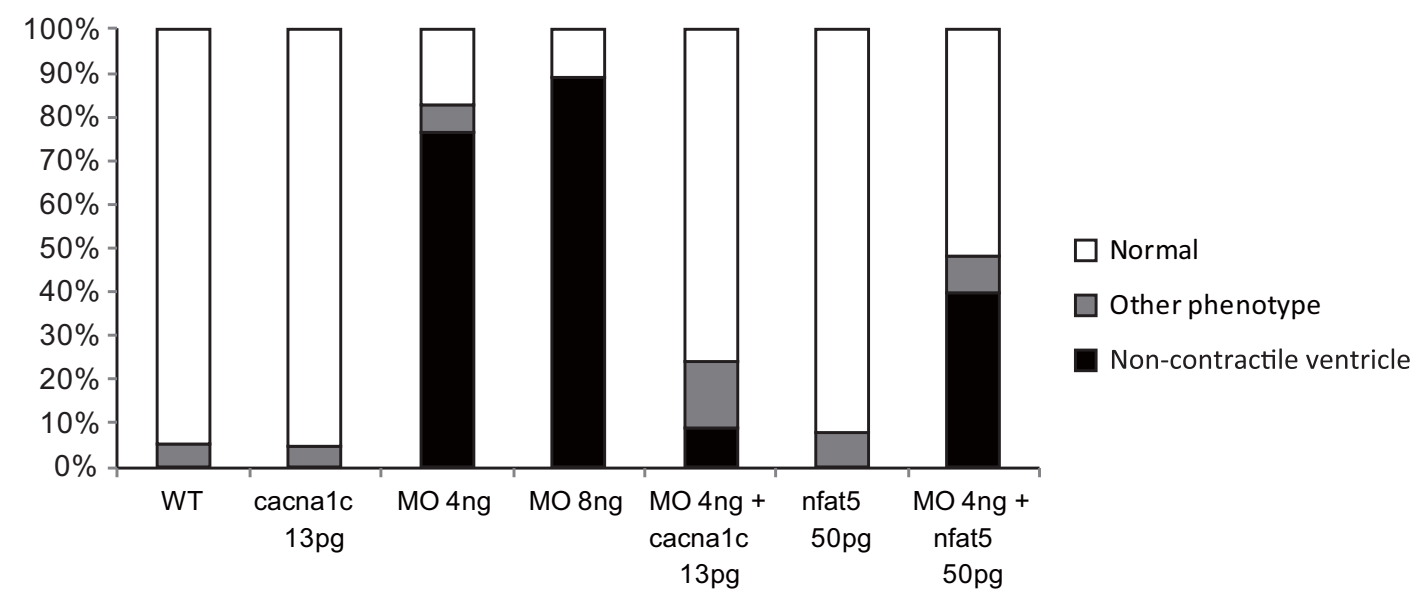

